\title{
Tradução para o português do Brasil e adaptação cultural do Sensory Profile
}

\author{
Jací Carnicelli Mattos' \\ Maria Eloisa Famá D’Antino \\ Roberta Monterazzo Cysneiros \\ Universidade Presbiteriana Mackenzie, São Paulo - SP - Brasil
}

\begin{abstract}
Resumo: Considerando o fato de não haver instrumentos brasileiros para avaliar habilidades do processamento sensorial e seus efeitos na funcionalidade de crianças e adolescentes, este estudo traduziu para o português do Brasil e adaptou culturalmente o Sensory Profile. O estudo se encaixa num modelo de pesquisa não experimental, transversal e descritiva, e os passos foram os seguintes: tradução, retrotradução, revisão por um comitê de especialistas, avaliação das pontuações e verificação da consistência interna do instrumento. A amostra foi composta de 50 pais de crianças de 5 a 10 anos de idade, regularmente matriculadas numa escola particular de ensino fundamental da cidade de São Paulo. A aplicação do questionário traduzido e adaptado permitiu a verificação de sua consistência interna em 0,76 , o que sugere que o instrumento é compreensível e apropriado para estudos futuros em que se busquem evidências de validade.
\end{abstract}

Palavras-chave: Sensory Profile; tradução; adaptação cultural; processamento sensorial; crianças.

\section{TRANSLATION INTO BRAZILIAN PORTUGUESE AND CULTURAL ADAPTATION OF THE SENSORY PROFILE}

\begin{abstract}
Considering that there are not any Brazilian instruments to evaluate sensory processing abilities and their effect on the functionality of children and teenagers, this study translated into Brazilian Portuguese and culturally adapted the Sensory Profile. This study fits is a non-experimental, cross-sectional and descriptive research, and the steps were: translation, back-translation, revision by a specialist committee on analysis, evaluation of the scoring and verification of the instrument's internal consistency. The sample consisted of 50 parents of children aged 5 to 10 , regularly enrolled on a private elementary school in the city of São Paulo, Brazil. The application of the translated and culturally adapted questionnaire yielded an internal consistency of 0.76 , suggesting that the translated and adapted instrument is understandable and suited for future studies directed toward obtaining evidence of validity.
\end{abstract}

Keywords: Sensory Profile; translation; cultural adaptation; sensory processing; child.

\section{TRADUCCIÓN AL PORTUGUÉS DE BRASIL Y ADAPTACIÓN CULTURAL DEL SENSORY PROFILE}

Resumen: Teniendo en cuenta el hecho de que no existen instrumentos brasileños para evaluar las habilidades de procesamiento sensorial y sus efectos en la funcionalidad

${ }^{1}$ Endereço para correspondência: Jací Carnicelli Mattos, Programa de Pós-Graduação em Distúrbios do Desenvolvimento, Universidade Presbiteriana Mackenzie, Rua da Consolação, 930, Prédio 28, São Paulo - SP Brasil. CEP: 01302-907. E-mail: jcarnicellimattos@gmail.com. 


\begin{abstract}
de los niños y adolescentes, este estudio tradujo al portugués de Brasil y adaptó culturalmente el Sensory Profile. El estudio se inscribe en un modelo de investigación no experimental, transversal, descriptiva y los pasos fueron los siguientes: la traducción, retro-traducción, la revisión por parte de un comité de expertos, la evaluación de los resultados y la verificación de la consistencia interna del instrumento. La muestra estuvo formada por 50 padres de niños de 5 a 10 años de edad, matriculados regularmente en una escuela primaria privada en São Paulo. La aplicación del cuestionario traducido y adaptado permitió comprobar su consistencia interna de 0,76 , lo que sugiere que el instrumento es comprensible y adecuado para futuros estudios que buscan evidencia de validez.
\end{abstract}

Palabras clave: Sensory Profile; traducción; adaptación cultural; procesamiento sensorial; niños.

A Teoria da Integração Sensorial, descrita por Anne Jean Ayres (1972), é definida como um processo neurológico no qual as informações sensoriais oriundas do meio ambiente são registradas, moduladas, organizadas e interpretadas para serem posteriormente utilizadas em resposta às diferentes demandas ambientais. O Transtorno do Processamento Sensorial (TPS) tem sido observado em inúmeras condições, como Transtornos do Espectro Autista (TEA) (Tomcheck \& Dunn, 2007; Ashburner, Ziviani, \& Rodger, 2008; Brown, Leo, \& Austin, 2008; Brown \& Dunn, 2010; Case-Smith, Weaver, \& Fristad, 2015), Transtorno do Déficit de Atenção e Hiperatividade (TDAH) (Koziol \& Budding, 2012) e esquizofrenia (Javitt, 2009), comprometendo, em graus variáveis, as atividades sociais, a aprendizagem e o comportamento socioemocional no cotidiano. Para exemplificar a importância da investigação no processamento sensorial, estima-se que mais de $80 \%$ dos indivíduos com o TEA apresentem problemas com o processamento sensorial, de tal maneira que a hiper ou hiporreatividade a estímulos sensoriais ou o interesse incomum por aspectos sensoriais do ambiente foram inseridos nos critérios diagnósticos para TEA na quinta edição do Manual diagnóstico e estatístico de transtornos mentais (DSM-5) da American Psychiatry Association (Case-Smith et al., 2015). Nas últimas décadas, o interesse na investigação desse campo cresceu significativamente e impulsionou o desenvolvimento de instrumentos para avaliação e mensuração do perfil sensorial, de forma a propiciar intervenções direcionadas às necessidades individuais. Diversos instrumentos padronizados para avaliação e mensuração do processamento motor e sensorial e de dificuldades socioemocionais estão disponíveis, a saber:

- Sensory Integration and Praxis Tests (Ayres, 1989): instrumento de avaliação sensorial composto por 17 subtestes para a faixa etária de 4 a 8 anos e direcionado a pais e cuidadores.

- Test of Sensory Functions in Infants (DeGangi \& Stanley, 1989): questionário com 24 perguntas para lactentes de 4 a 18 meses de idade e direcionado a pais ou cuidadores.

- Sensory Profile (Dunn, 1999b): questionário com 125 perguntas para a faixa etária de 3 a 10 anos e direcionado a pais ou cuidadores.

- Short Sensory Profile (Dunn, 1999b): versão resumida do Sensory Profile com 38 questões para a faixa etária de 3 a 10 anos e direcionada a pais ou cuidadores. 
- Infant Toddler Sensory Profile (Dunn, 2002): questionário com 36 perguntas para lactentes de 0 a 6 meses de idade e 48 perguntas para lactentes de 7 a 36 meses; direcionado a pais ou cuidadores.

- Adolescent/Adult Sensory Profile (Dunn, 2002): questionário de autopreenchimento com 60 itens para a faixa etária a partir de 11 anos.

- Sensory Profile School Companion (Dunn, 2006): questionário com 62 perguntas para a faixa etária de 3 a 11 anos e direcionado a professores.

O Sensory Profile (Dunn, 1999a), desenvolvido por Winnie Dunn em 1994, foi concebido para avaliar e mensurar quanto o processamento sensorial facilita ou dificulta o desempenho funcional em tarefas diárias, com vistas a contribuir para o planejamento de intervenções (Dunn, 1999b). O instrumento original era composto de 99 questões, e a pesquisa envolveu a participação de 64 pais de crianças com desenvolvimento típico, com idades entre 3 e 10 anos. Pesquisas posteriores (Dunn \& Brown, 1997; Kientz \& Dunn, 1997; Ermer \& Dunn, 1997) possibilitaram o aperfeiçoamento do instrumento, e o número de questões foi ampliado para 125. As pesquisas realizadas para o desenvolvimento e aperfeiçoamento do instrumento ocorreram entre 1993 e 1999, e envolveram 155 terapeutas ocupacionais, 1.037 crianças com desenvolvimento típico, 61 com TDAH, 32 com TEA, 24 com síndrome do X Frágil e 21 com Transtorno Modular Sensorial com idades entre 3 e 14 anos (Dunn, 1999b). O instrumento avalia, de forma detalhada, o processamento sensorial, é apropriado para ser utilizado em programas de rastreamento e protocolos de pesquisa, e está organizado em três áreas: processamento sensorial, área subdividida em seis categorias (audição, visão, movimento, tato, multissensorial e oral); modulação, área subdividida em cinco categorias (tônus, posição corporal/movimento, nível de atividade, respostas emocionais e estímulo visual); e comportamento e respostas emocionais, área subdividida em três categorias (emocional/social, efeitos comportamentais no processamento sensorial e limiares para respostas). Os benefícios da utilização do instrumento são: resgatar informações importantes sobre o processamento sensorial, associar claramente o processamento sensorial com o desempenho cotidiano, fornecer informações para a tomada de decisões a partir de uma base teórica, envolver os cuidadores como membros críticos da equipe que convive/trabalha com a criança/adolescente, ser aplicável a crianças com todo tipo de deficiência e níveis de gravidade, ser simples quanto à aplicação, pontuação e interpretação (Dunn, 1999b).

Considerando a robustez do Sensory Profile, a tradução, a adaptação cultural e a busca por evidências de validade foram realizadas em vários idiomas e em diferentes culturas. Neuman, Greenberg, Labovitz e Suzuki (2004) realizaram a tradução e adaptação cultural do Sensory Profile do inglês para o hebraico utilizando a versão hebraica do Short Sensory Profile. Neuman (2006) comparou os escores médios das crianças israelenses com desenvolvimento típico com aqueles já publicados para as crianças norte-americanas. Apesar de as crianças israelenses apresentarem, no geral, pontuações menores, elas estavam dentro da faixa estabelecida para o desempenho típico. Nos 
anos subsequentes, evidências de validade da versão hebraica do instrumento foram obtidas por meio de pesquisas com diversos grupos clínicos: dermatite atópica (Engel-Yeger et al., 2007, Engel-Yeger, Hardal-Nasser, \& Gal, 2011), TDAH (Koziol \& Budding, 2015), deficiência intelectual (Engel-Yeger et al., 2011) e asma (Engel-Yeger, Almog, \& Kessel, 2014). Engel-Yeger (2010) também investigou a aplicabilidade do instrumento para o rastreio do TPS em crianças israelenses com desenvolvimento típico, bem como para avaliar a relação entre o TPS e os parâmetros sociodemográficos. Brown et al. (2008) demonstraram a validade discriminativa do Sensory Profile por meio da comparação dos escores do processamento sensorial de crianças australianas com TEA e com desenvolvimento típico pareadas por sexo e idade. Chung (2006) demonstrou evidências de validade psicométricas da versão chinesa do Adult Sensory Profile em participantes com função cognitiva normal e demência. Almomani, Brown, Dahab, Almomani e Nadar (2014) demonstraram a adequação da tradução e adaptação transcultural, bem como evidências de validade psicométricas da versão arábica do Adolescent/Adult Sensory Profile, em participantes adultos bilíngues em árabe e inglês. Ganapathy e Priyadarshini (2014) demonstraram evidências de validade psicométricas da versão tâmil do Short Sensory Profile em crianças com desenvolvimento típico e TPS pareadas por sexo e idade.

Com base na ampla gama de utilização do Sensory Profile e na inexistência de instrumentos padronizados para a realidade brasileira, este estudo teve como objetivo realizar a tradução e adaptação transcultural para o português do Brasil do Sensory Profile (Dunn, 1999a).

\section{Método}

O presente estudo encaixa-se no modelo de pesquisa não experimental (baseada em categorias, conceitos, contextos ou acontecimentos já ocorridos), transversal (coleta única de dados) e descritiva (reporta os dados sobre categorias, conceitos, contextos ou acontecimentos e especifica características e perfis de pessoas, grupos ou comunidades), segundo Sampieri, Collado e Lucio (2006).

O estudo foi submetido à apreciação do Comitê de Ética em Pesquisa da Universidade Presbiteriana Mackenzie (UPM) e aprovado por meio do Parecer Consubstanciado n. 836.568. O Termo de Consentimento Livre e Esclarecido foi também aprovado por esse comitê e apresentado aos participantes (pais de alunos de 5 a 10 anos de idade) e à direção da escola para leitura e assinatura antes da aplicação do instrumento.

\section{Tradução e adaptação cultural}

Para a realização do projeto, foi firmado um acordo - para a tradução e adaptação cultural do instrumento Sensory Profile (Dunn, 1999b) - entre a Editora Pearson, que detém os direitos autorais do instrumento, e a UPM.

Os procedimentos adotados para a tradução e adaptação cultural seguiram o método proposto por Alexandre \& Guirardello (2002): 
- A tradução foi realizada por tradutora independente com formação em Psicologia, mestra e doutoranda nas áreas de saúde e educação, tendo como idioma nativo o português do Brasil e sendo fluente no idioma inglês.

- A retrotradução foi realizada por outro tradutor independente, tendo como idioma nativo o inglês e sendo fluente no português do Brasil. Esse profissional não teve contato com o instrumento original e concluiu o trabalho com base na tradução da profissional supracitada.

- A revisão da tradução foi realizada por um comitê composto pelas autoras deste estudo e por uma terapeuta ocupacional. Analisaram-se e compararam-se todas as questões da versão original com as da tradução e retrotradução. Das 125 questões, 40 foram reformuladas com o objetivo de alcançar uma equivalência semântica (de significado), idiomática (de uso de expressões), conceitual (de coerência dos itens) e cultural (de adequação ao contexto da nossa cultura) entre o inglês e o português brasileiro, e, assim, tornar as referidas questões mais compreensíveis para os participantes/respondentes.

- A versão final, obtida após inseridas as reformulações do comitê de especialistas, foi aplicada aos cuidadores a fim de verificar a compreensibilidade das questões do instrumento por parte dos respondentes.

- A avaliação dos valores das pontuações foi realizada a partir de instruções do manual do instrumento, e obteve-se a verificação da consistência interna pelo alfa de Cronbach, por meio do cálculo da variância das categorias e da variância dos escores brutos.

\section{Verificação da aplicabilidade e consistência interna}

\section{Participantes}

Participaram deste estudo 47 crianças com desenvolvimento típico, duas com síndrome de Down e uma com TEA, com idades entre 5 e 10 anos, regularmente matriculadas numa escola particular de ensino fundamental da cidade de São Paulo.

As informações sobre o diagnóstico dos participantes foram fornecidas pelos pais/ cuidadores em conformidade com os dados relatados pela coordenadora pedagógica da instituição.

Em relação aos respondentes (pais/cuidadores), 92\% eram do sexo feminino e $8 \%$ do sexo masculino. A faixa etária variou de 30 a 63 anos, com a média de 41,2 \pm 4,9 anos. O grau de parentesco com a criança foi o seguinte: $90 \%$ de mães, $8 \%$ de pais e $2 \%$ de avós. Quanto à escolaridade, $10 \%$ possuíam o ensino médio completo; $66 \%$, ensino superior completo; $6 \%$, ensino superior incompleto; e 18\%, mestrado ou doutorado. O critério de inclusão para a participação da amostra consistiu em ser um dos responsáveis pela criança e conviver diariamente com ela.

Os sujeitos com síndrome de Down e TEA foram incluídos como participantes considerando que a amostra é de conveniência e os respondentes preenchiam os critérios de inclusão. 
O instrumento foi aplicado aos pais/cuidadores de crianças com idade de 5 a 10 anos, muito embora o instrumento seja destinado à faixa etária de 3 a 10 anos. A restrição da faixa etária deveu-se ao fato de as crianças com 3 e 4 anos apresentarem comportamentos e respostas diferentes em relação às mais velhas, especialmente em algumas categorias/seções, como respostas emocionais, percepção motora fina e outras, conforme consta no manual do Sensory Profile.

Como descrito no capítulo 4 do manual do Sensory Profile, há três maneiras de aplicar o instrumento. A primeira é enviar o questionário ao cuidador/responsável pela criança com uma carta, na qual devem constar as instruções, o objetivo do instrumento e um contato telefônico caso surja alguma dúvida no preenchimento e nas respostas às questões. A segunda forma é aplicar o questionário na clínica ou outro local em que a criança esteja sendo avaliada (enquanto a criança é submetida à avaliação, o cuidador/responsável responde ao instrumento). A terceira maneira é acompanhar pessoalmente o preenchimento do questionário e ajudar o cuidador/responsável se ele apresentar alguma dificuldade em relação à linguagem do instrumento ou à leitura e interpretação das questões (Dunn, 1999b). Decidiu-se pela terceira forma, e, assim, os participantes foram acompanhados e orientados pessoalmente pelas pesquisadoras. A média de tempo por participante para o preenchimento do cabeçalho e das respostas às questões foi de 30 minutos.

\section{Avaliação dos valores das pontuações}

As pontuações para cada uma das questões foram obtidas com base nas instruções do Capítulo 4 do manual do Sensory Profile, e utilizou-se como referência a Folha Resumo de Pontuação do instrumento em sua versão original (Dunn, 1999b): "sempre" (um ponto), "frequentemente" (dois pontos), "ocasionalmente" (três pontos), "raramente" (quatro pontos) e "nunca" (cinco pontos).

A partir da pontuação dada a cada uma das 125 questões para cada um dos 50 participantes, os subtotais das 14 seções/categorias distribuídas nas três áreas do Sensory Profile (processamento sensorial, modulação e comportamento e respostas emocionais) foram utilizados para verificação da consistência do instrumento. O grau de consistência interna foi mensurado pelo alfa de Cronbach, empregando a versão comercial 17.0 do programa (Statistical Package for Social Sciences).

\section{Resultados}

\section{Tradução e adaptação cultural}

Num estudo de tradução e adaptação cultural de instrumentos de avaliação, algumas etapas devem ser criteriosamente desenvolvidas. A análise e comparação entre a versão original, a tradução e a retrotradução é uma delas. Várias palavras ou expressões presentes na tradução e na retrotradução foram alteradas para adequação 
cultural de significados, usos e coerência (Quadro 1). Das 125 questões, 40 (32\%) foram modificadas para se obter uma equivalência semântica, idiomática, conceitual e cultural entre o inglês e o português brasileiro. Durante a aplicação, apenas três (6\%) dos 50 respondentes fizeram observações em relação às questões nas quais aparece a palavra não à frente de uma ação (por exemplo: não gosta de andar de carro), comentando que o vocábulo, nessas situações, pode criar uma dificuldade na marcação da frequência com que o comportamento é apresentado pela criança (sempre, frequentemente, ocasionalmente, raramente, nunca).

\section{Quadro I. Avaliação da equivalência semântica, idiomática, conceitual e cultural entre o Sensory Profile original, a tradução, a retrotradução e a versão para aplicação (análise e comparação pelo comitê de especialistas)}

\begin{tabular}{|c|c|c|c|}
\hline Original & Tradução & Retrotradução & Versão para aplicação \\
\hline $\begin{array}{l}\text { 3. Has trouble } \\
\text { completing tasks when } \\
\text { the radio is on. }\end{array}$ & $\begin{array}{l}\text { 3. Tem dificuldade em } \\
\text { completar tarefas quando } \\
\text { o rádio está ligado. }\end{array}$ & $\begin{array}{l}\text { 3. Has difficulty } \\
\text { completing tasks when } \\
\text { a radio is playing. }\end{array}$ & $\begin{array}{l}\text { 3. Tem problemas para } \\
\text { completar atividades } \\
\text { quando o rádio está ligado. }\end{array}$ \\
\hline $\begin{array}{l}\text { 4. Is distracted or has } \\
\text { trouble functioning if } \\
\text { there is a lot of noise } \\
\text { around. }\end{array}$ & $\begin{array}{l}\text { 4. Distrai-se ou tem } \\
\text { dificuldade em realizar } \\
\text { atividades quando há } \\
\text { muito barulho no } \\
\text { ambiente. }\end{array}$ & $\begin{array}{l}\text { 4. Is easily distracted and } \\
\text { has difficulty performing } \\
\text { activities in noisy } \\
\text { surroundings. }\end{array}$ & $\begin{array}{l}\text { 4. Distrai-se ou tem } \\
\text { problemas em realizar } \\
\text { atividades quando há } \\
\text { muito barulho no } \\
\text { ambiente. }\end{array}$ \\
\hline $\begin{array}{l}\text { 6. Appears to not hear } \\
\text { what you say (for example, } \\
\text { does not "tune-in" to } \\
\text { what you say, appears to } \\
\text { ignore you). }\end{array}$ & $\begin{array}{l}\text { 6. Parece não ouvir o que } \\
\text { é dito (por exemplo, não } \\
\text { está “atento” ao que é } \\
\text { dito, parece ignorar). }\end{array}$ & $\begin{array}{l}\text { 6. Does not seem to } \\
\text { hear what is said (for } \\
\text { example, does not pay } \\
\text { attention to and seems } \\
\text { to ignore what is said). }\end{array}$ & $\begin{array}{l}\text { 6. Parece não ouvir o que } \\
\text { é falado (por exemplo, não } \\
\text { presta atenção ao que é } \\
\text { dito, parece ignorar). }\end{array}$ \\
\hline $\begin{array}{l}\text { I0. Expresses discomfort } \\
\text { or avoids bright light } \\
\text { (for example, hides from } \\
\text { with sunlight through } \\
\text { window in car). }\end{array}$ & $\begin{array}{l}\text { I0. Expressa desconforto } \\
\text { com ou evita luzes fortes } \\
\text { (por exemplo, esconde-se } \\
\text { da luz do sol que entra } \\
\text { pela janela do carro). }\end{array}$ & $\begin{array}{l}\text { I0. Expresses discomfort } \\
\text { with or avoids strong light } \\
\text { (for example, hides from } \\
\text { sunlight coming in through } \\
\text { the car window). }\end{array}$ & $\begin{array}{l}\text { I0. Incomoda-se com ou } \\
\text { evita luzes fortes (por } \\
\text { exemplo, esconde-se da } \\
\text { luz do sol que entra pela } \\
\text { janela do carro). }\end{array}$ \\
\hline $\begin{array}{l}\text { I2. Becomes frustrated } \\
\text { when trying to find } \\
\text { objects in competing } \\
\text { backgrounds (for } \\
\text { example a cluttered } \\
\text { drawer). }\end{array}$ & $\begin{array}{l}\text { 12. Frustra-se quando } \\
\text { tenta encontrar objetos } \\
\text { em fundos confusos (por } \\
\text { exemplo, em uma gaveta } \\
\text { desarrumada). }\end{array}$ & $\begin{array}{l}\text { 12. Becomes frustrated } \\
\text { when trying to find } \\
\text { objects in confused } \\
\text { environments (for } \\
\text { example a messy } \\
\text { drawer). }\end{array}$ & $\begin{array}{l}\text { I2. Frustra-se quando } \\
\text { tenta encontrar objetos } \\
\text { em ambientes } \\
\text { desorganizados (por } \\
\text { exemplo, em uma gaveta } \\
\text { desarrumada). }\end{array}$ \\
\hline $\begin{array}{l}\text { l4. Is bothered by bright } \\
\text { lights after others have } \\
\text { adapted to the light. }\end{array}$ & $\begin{array}{l}\text { I4. Incomoda-se com } \\
\text { luzes fortes mesmo } \\
\text { quando outros já tenham } \\
\text { se adaptado. }\end{array}$ & $\begin{array}{l}\text { I4. Is bothered by strong } \\
\text { lights, even when others } \\
\text { have already gotten used } \\
\text { to them. }\end{array}$ & $\begin{array}{l}\text { I4. Incomoda-se com } \\
\text { luzes fortes mesmo } \\
\text { quando outras pessoas já } \\
\text { se adaptaram. }\end{array}$ \\
\hline
\end{tabular}




\section{Quadro I. Avaliação da equivalência semântica, idiomática, conceitual e cultural entre o Sensory Profile original, a tradução, a retrotradução e a versão para aplicação (análise e comparação pelo comitê de especialistas) (continuação)}

\begin{tabular}{|c|c|c|c|}
\hline Original & Tradução & Retrotradução & Versão para aplicação \\
\hline $\begin{array}{l}\text { 16. Looks carefully or } \\
\text { intensely at objects/people } \\
\text { (for example, stares). }\end{array}$ & $\begin{array}{l}\text { 16. Olha cuidadosa ou } \\
\text { intensamente para objetos/ } \\
\text { pessoas (olha fixamente). }\end{array}$ & $\begin{array}{l}\text { 16. Looks carefully or } \\
\text { intensely at objects/ } \\
\text { people (stares). }\end{array}$ & $\begin{array}{l}\text { 16. Olha para partes } \\
\text { específicas de pessoas e } \\
\text { objetos por muito tempo. }\end{array}$ \\
\hline $\begin{array}{l}\text { 17. Has a hard time } \\
\text { finding objects in } \\
\text { competing backgrounds } \\
\text { (for example, shoes in a } \\
\text { messy room, favorite toy } \\
\text { in the "junk drawer"). }\end{array}$ & $\begin{array}{l}\text { I7. Tem dificuldade em } \\
\text { encontrar objetos em } \\
\text { fundos confusos (por } \\
\text { exemplo, sapatos em um } \\
\text { quarto desarrumado, o } \\
\text { brinquedo favorito na } \\
\text { "gaveta da bagunça"). }\end{array}$ & $\begin{array}{l}\text { 17. Finds it difficult to } \\
\text { find objects in confused } \\
\text { environments (for } \\
\text { example, shoes in a } \\
\text { messy room, a favorite } \\
\text { toy in a "junk drawer"). }\end{array}$ & $\begin{array}{l}\text { 17. Tem dificuldade em } \\
\text { encontrar objetos em } \\
\text { ambientes desorganizados } \\
\text { (por exemplo, sapatos em } \\
\text { um quarto desarrumado, } \\
\text { o brinquedo favorito na } \\
\text { "gaveta da bagunça"). }\end{array}$ \\
\hline $\begin{array}{l}\text { 19. Dislikes activities } \\
\text { where head is upside } \\
\text { down (for example, } \\
\text { somersaults, } \\
\text { roughhousing). }\end{array}$ & $\begin{array}{l}\text { 19. Não gosta de } \\
\text { atividades em que fique } \\
\text { de ponta-cabeça (por } \\
\text { exemplo, cambalhotas, } \\
\text { brincar de cavalinho). }\end{array}$ & $\begin{array}{l}\text { 19. Does not like } \\
\text { upside-down activities } \\
\text { (for example, } \\
\text { roughhousing, tumbling). }\end{array}$ & $\begin{array}{l}\text { 19. Não gosta de } \\
\text { atividades em que fique } \\
\text { de ponta-cabeça (por } \\
\text { exemplo, cambalhotas). }\end{array}$ \\
\hline $\begin{array}{l}\text { 20. Avoids playground } \\
\text { equipment or moving } \\
\text { toys (for example, swing } \\
\text { set, merry-go-round). }\end{array}$ & $\begin{array}{l}\text { 20. Evita os brinquedos do } \\
\text { playground ou brinquedos } \\
\text { que se movimentam } \\
\text { (por exemplo, balanço } \\
\text { ou carrossel). }\end{array}$ & $\begin{array}{l}\text { 20. Avoids playground } \\
\text { toys or moving toys (for } \\
\text { example, swings or } \\
\text { merry-go-round). }\end{array}$ & $\begin{array}{l}\text { 20. Evita os brinquedos do } \\
\text { parquinho ou brinquedos } \\
\text { que se movimentam (por } \\
\text { exemplo, balanço ou } \\
\text { carrossel). }\end{array}$ \\
\hline $\begin{array}{l}\text { 22. Holds head upright, } \\
\text { even when bending over } \\
\text { or leaning (for example, } \\
\text { maintains a rigid position/ } \\
\text { posture during activities). }\end{array}$ & $\begin{array}{l}\text { 22. Endireita a cabeça } \\
\text { mesmo quando está se } \\
\text { inclinando ou apoiando } \\
\text { (por exemplo, mantém } \\
\text { uma posição/postura } \\
\text { rígida durante atividades). }\end{array}$ & $\begin{array}{l}\text { 22. Holds head up } \\
\text { straight even when } \\
\text { bending over or leaning } \\
\text { (for example, maintains } \\
\text { a rigid position/posture } \\
\text { during activities). }\end{array}$ & $\begin{array}{l}\text { 22. Mantém a cabeça ereta } \\
\text { quando está se inclinando } \\
\text { ou se curvando (por } \\
\text { exemplo, mantém uma } \\
\text { posição/postura rígida } \\
\text { durante atividades). }\end{array}$ \\
\hline $\begin{array}{l}\text { 24. Seeks all kinds of } \\
\text { movement and this } \\
\text { interferes with daily } \\
\text { routines (for example, } \\
\text { can't sit still, fidgets). }\end{array}$ & $\begin{array}{l}\text { 24. Procura todo tipo } \\
\text { de movimento e isso } \\
\text { atrapalha sua rotina diária } \\
\text { (por exemplo, não } \\
\text { consegue sentar quieto, } \\
\text { fica irrequieto). }\end{array}$ & $\begin{array}{l}24 \text {. Seeks any type of } \\
\text { movement, making a } \\
\text { daily routine difficult (for } \\
\text { example, cannot sit still, } \\
\text { is restless). }\end{array}$ & $\begin{array}{l}\text { 24. Procura todo tipo de } \\
\text { movimento e isso } \\
\text { atrapalha sua rotina diária } \\
\text { (por exemplo, não } \\
\text { consegue permanecer } \\
\text { sentado, fica irrequieto). }\end{array}$ \\
\hline $\begin{array}{l}\text { 25. Seeks out all kinds } \\
\text { of movement activities } \\
\text { (for example, being } \\
\text { whirled by adult, } \\
\text { merry-go-rounds, } \\
\text { playground equipment, } \\
\text { moving toys). }\end{array}$ & $\begin{array}{l}\text { 25. Procura todo tipo de } \\
\text { atividade com movimento } \\
\text { (por exemplo, ser girado } \\
\text { por um adulto, carrossel, } \\
\text { brinquedos de playground, } \\
\text { brinquedos que se } \\
\text { movimentam). }\end{array}$ & $\begin{array}{l}25 . \text { Seeks any type of } \\
\text { activity with movement } \\
\text { (for example, being } \\
\text { twirled around by an } \\
\text { adult, merry-go-round, } \\
\text { playground toys, toys } \\
\text { which move). }\end{array}$ & $\begin{array}{l}\text { 25. Procura todo tipo de } \\
\text { atividade com movimento } \\
\text { (por exemplo, ser girado } \\
\text { por um adulto, carrossel, } \\
\text { brinquedos de parquinho, } \\
\text { brinquedos que se } \\
\text { movimentam). }\end{array}$ \\
\hline
\end{tabular}




\section{Quadro I. Avaliação da equivalência semântica, idiomática, conceitual e cultural entre o Sensory Profile original, a tradução, a retrotradução e a versão para aplicação (análise e comparação pelo comitê de especialistas) (continuação)}

\begin{tabular}{|c|c|c|c|}
\hline Original & Tradução & Retrotradução & Versão para aplicação \\
\hline $\begin{array}{l}\text { 27. Rocks unconsciously } \\
\text { (for example, while } \\
\text { watching TV). }\end{array}$ & $\begin{array}{l}\text { 27. Balança } \\
\text { inconscientemente } \\
\text { (por exemplo, enquanto } \\
\text { assiste à TV). }\end{array}$ & $\begin{array}{l}\text { 27. Sways unconsciously } \\
\text { (for example, while } \\
\text { watching TV). }\end{array}$ & $\begin{array}{l}\text { 27. Balança-se } \\
\text { involuntariamente (por } \\
\text { exemplo, enquanto assiste } \\
\text { à TV). }\end{array}$ \\
\hline $\begin{array}{l}\text { 28. Rocks in desk/chair/on } \\
\text { floor. }\end{array}$ & $\begin{array}{l}\text { 28. Balança na cadeira/ } \\
\text { carteira/chão. }\end{array}$ & $\begin{array}{l}\text { 28. Sways on the chair/ } \\
\text { desk/floor. }\end{array}$ & $\begin{array}{l}\text { 28. Balança-se na cadeira/ } \\
\text { carteira/chão. }\end{array}$ \\
\hline $\begin{array}{l}\text { 33. Is sensitive to certain } \\
\text { fabrics (for example, is } \\
\text { particular about certain } \\
\text { clothes or bedsheets). }\end{array}$ & $\begin{array}{l}\text { 33. É sensível a certos } \\
\text { tecidos (por exemplo, } \\
\text { é exigente com certos } \\
\text { tipos de roupa ou roupa } \\
\text { de cama). }\end{array}$ & $\begin{array}{l}\text { 33. Is sensitive to certain } \\
\text { fabrics (for example, is } \\
\text { demanding of certain } \\
\text { types of clothing or } \\
\text { bedding). }\end{array}$ & $\begin{array}{l}\text { 33. É sensível a certos } \\
\text { tecidos (por exemplo, } \\
\text { reclama de certos tipos de } \\
\text { roupa ou roupa de cama). }\end{array}$ \\
\hline $\begin{array}{l}\text { 39. Rubs or scratches } \\
\text { out a spot that has been } \\
\text { touched. }\end{array}$ & $\begin{array}{l}\text { 39. Esfrega ou coça } \\
\text { um ponto que tenha } \\
\text { sido tocado. }\end{array}$ & $\begin{array}{l}\text { 39. Rubs or scratches } \\
\text { a spot that has been } \\
\text { touched. }\end{array}$ & $\begin{array}{l}\text { 39. Esfrega ou coça uma } \\
\text { parte do corpo que tenha } \\
\text { sido tocada. }\end{array}$ \\
\hline $\begin{array}{l}\text { 42. Decreased awareness } \\
\text { of pain and temperature. }\end{array}$ & $\begin{array}{l}\text { 42. Tem reduzida } \\
\text { percepção para dor } \\
\text { e temperatura. }\end{array}$ & $\begin{array}{l}\text { 42. Has a reduced } \\
\text { perception of pain } \\
\text { and temperature. }\end{array}$ & $\begin{array}{l}\text { 42. Parece não sentir } \\
\text { dor ou alterações de } \\
\text { temperatura. }\end{array}$ \\
\hline $\begin{array}{l}\text { 49. Looks away from } \\
\text { tasks to notice all actions } \\
\text { in the room. }\end{array}$ & $\begin{array}{l}\text { 49. Desvia o olhar das } \\
\text { tarefas para observar } \\
\text { todas as ações ao redor. }\end{array}$ & $\begin{array}{l}\text { 49. Looks away from } \\
\text { tasks to watch what is } \\
\text { going on around him. }\end{array}$ & $\begin{array}{l}\text { 49. Desvia o olhar das } \\
\text { atividades para observar } \\
\text { tudo o que acontece ao } \\
\text { redor. }\end{array}$ \\
\hline $\begin{array}{l}\text { 43. Doesn't seem to } \\
\text { notice when someone } \\
\text { touches arm or back } \\
\text { (for example, unaware). }\end{array}$ & $\begin{array}{l}\text { 43. Não parece sentir } \\
\text { quando alguém toca } \\
\text { seu braço ou costas } \\
\text { (por exemplo, quando } \\
\text { não percebe). }\end{array}$ & $\begin{array}{l}\text { 43. Does not seem to } \\
\text { feel it when someone } \\
\text { touches arm or back (for } \\
\text { example, doesn't notice). }\end{array}$ & $\begin{array}{l}\text { 43. Parece não perceber } \\
\text { quando alguém toca seu } \\
\text { braço ou costas. }\end{array}$ \\
\hline $\begin{array}{l}\text { 50. Seems oblivious within } \\
\text { an active environment } \\
\text { (for example, unaware } \\
\text { of activity). }\end{array}$ & $\begin{array}{l}\text { 50. Parece distraído } \\
\text { em ambientes ativos } \\
\text { (por exemplo, não } \\
\text { percebe as atividades). }\end{array}$ & $\begin{array}{l}\text { 50. Seems distracted } \\
\text { in busy environments } \\
\text { (for example, does not } \\
\text { notice activities). }\end{array}$ & $\begin{array}{l}\text { 50. Parece distraído em } \\
\text { ambiente ativo (por } \\
\text { exemplo, não percebe } \\
\text { as atividades). }\end{array}$ \\
\hline $\begin{array}{l}\text { 53. Leaves clothing } \\
\text { twisted on body. }\end{array}$ & $\begin{array}{l}\text { 53. Deixa a roupa } \\
\text { torcida no corpo. }\end{array}$ & $\begin{array}{l}\text { 53. Leaves clothes } \\
\text { twisted while wearing } \\
\text { them. }\end{array}$ & $\begin{array}{l}\text { 53. Deixa a roupa torcida } \\
\text { ou enrolada no corpo. }\end{array}$ \\
\hline $\begin{array}{l}\text { 54. Gags easily with food } \\
\text { texture or food utensils } \\
\text { in mouth. }\end{array}$ & $\begin{array}{l}\text { 54. Costuma sentir } \\
\text { náusea com texturas } \\
\text { ou utensílios de comida } \\
\text { na boca. }\end{array}$ & $\begin{array}{l}\text { 54. Feels nauseous from } \\
\text { the texture of food or } \\
\text { utensils in mouth. }\end{array}$ & $\begin{array}{l}\text { 54. Costuma ter ânsia de } \\
\text { vômito com determinadas } \\
\text { texturas de alimentos ou } \\
\text { talheres quando levados } \\
\text { à boca. }\end{array}$ \\
\hline
\end{tabular}




\section{Quadro I. Avaliação da equivalência semântica, idiomática, conceitual e cultural entre o Sensory Profile original, a tradução, a retrotradução e a versão para aplicação (análise e comparação pelo comitê de especialistas) (continuação)}

\begin{tabular}{|c|c|c|c|}
\hline Original & Tradução & Retrotradução & Versão para aplicação \\
\hline $\begin{array}{l}\text { 55. Avoids certain tastes } \\
\text { or food smells that are } \\
\text { typically part of children's } \\
\text { diets. }\end{array}$ & $\begin{array}{l}\text { 55. Evita certos sabores } \\
\text { ou cheiros de comida que } \\
\text { são tipicamente parte da } \\
\text { dieta infantil. }\end{array}$ & $\begin{array}{l}\text { 55. Avoids certain food } \\
\text { flavors or aromas which } \\
\text { are commonly present } \\
\text { in children's foods. }\end{array}$ & $\begin{array}{l}\text { 55. Evita certos sabores } \\
\text { ou cheiros de alimentos } \\
\text { para crianças. }\end{array}$ \\
\hline $\begin{array}{l}\text { 56. Will only eat certain } \\
\text { tastes (list: }\end{array}$ & $\begin{array}{l}\text { 56. Ingere somente } \\
\text { certos sabores } \\
\text { (listar:__ }) \text {. }\end{array}$ & $\begin{array}{l}\text { 56. Only eats foods } \\
\text { of certain flavors } \\
\text { (list: }\end{array}$ & $\begin{array}{l}\text { 56. Come somente } \\
\text { alimentos que têm certos } \\
\text { sabores (listar: }\end{array}$ \\
\hline $\begin{array}{l}\text { 57. Limits self to } \\
\text { particular food } \\
\text { textures/ temperatures } \\
\text { (list:___ }\end{array}$ & $\begin{array}{l}\text { 57. Limita-se somente } \\
\text { a certas texturas/ } \\
\text { temperaturas de comida } \\
\text { (listar: }\end{array}$ & $\begin{array}{l}\text { 57. Only accepts foods } \\
\text { of certain textures/ } \\
\text { temperatures } \\
\text { (list: }\end{array}$ & $\begin{array}{l}\text { 57. Come somente } \\
\text { alimentos com certas } \\
\text { texturas/temperaturas } \\
\text { (listar: }\end{array}$ \\
\hline $\begin{array}{l}\text { 58. Picky eater, especially } \\
\text { regarding food textures. }\end{array}$ & $\begin{array}{l}\text { 58. É seletivo com comida, } \\
\text { especialmente em relação } \\
\text { a texturas. }\end{array}$ & $\begin{array}{l}\text { 58. Is selective about } \\
\text { food, especially in relation } \\
\text { to textures. }\end{array}$ & $\begin{array}{l}\text { 58. É seletivo com } \\
\text { alimentos, especialmente } \\
\text { em relação a texturas. }\end{array}$ \\
\hline $\begin{array}{l}\text { 62. Craves certain certain } \\
\text { foods (list: }\end{array}$ & $\begin{array}{l}\text { 62. Tem forte desejo por } \\
\text { certas comidas (listar: } \\
\text { ). }\end{array}$ & $\begin{array}{l}\text { 62. Has a strong desire } \\
\text { for certain foods } \\
\text { (list: }\end{array}$ & $\begin{array}{l}\text { 62. Tem forte desejo por } \\
\text { certos alimentos (listar: } \\
\text { ). }\end{array}$ \\
\hline 66. Moves stiffly. & $\begin{array}{l}\text { 66. Move-se com } \\
\text { rigidez. }\end{array}$ & $\begin{array}{l}\text { 66. Shows rigidity in } \\
\text { movements. }\end{array}$ & $\begin{array}{l}\text { 66. Movimenta-se com } \\
\text { rigidez. }\end{array}$ \\
\hline 70. Has a weak grasp. & $\begin{array}{l}\text { 70. Tem uma pegada } \\
\text { fraca. }\end{array}$ & 70. Has a weak grasp. & $\begin{array}{l}\text { 70. Tem preensão fraca } \\
\text { (não segura objetos com } \\
\text { firmeza). }\end{array}$ \\
\hline $\begin{array}{l}\text { 72. Props to support self } \\
\text { (even during activity). }\end{array}$ & $\begin{array}{l}\text { 72. Apoia-se (mesmo } \\
\text { durante atividade). }\end{array}$ & $\begin{array}{l}\text { 72. Leans on things } \\
\text { (even during an activity). }\end{array}$ & $\begin{array}{l}\text { 72. Busca segurar-se } \\
\text { ou apoiar-se em algo } \\
\text { para manter-se em pé } \\
\text { ou sentado (mesmo } \\
\text { durante atividade). }\end{array}$ \\
\hline $\begin{array}{l}\text { 76. Hesitates going up } \\
\text { or down curbs or steps } \\
\text { (for example, is cautious, } \\
\text { stops before moving). }\end{array}$ & $\begin{array}{l}\text { 76. Hesita em subir } \\
\text { ou descer guias ou } \\
\text { degraus (por exemplo, } \\
\text { é cauteloso, para antes } \\
\text { de movimentar-se). }\end{array}$ & $\begin{array}{l}\text { 76. Hesitates in going up } \\
\text { or down stairs or curbs } \\
\text { (for example, is cautious, } \\
\text { stops before proceeding). }\end{array}$ & $\begin{array}{l}\text { 76. Resiste em subir ou } \\
\text { descer guias ou degraus } \\
\text { (por exemplo, é cauteloso, } \\
\text { antes de iniciar um } \\
\text { movimento). }\end{array}$ \\
\hline $\begin{array}{l}\text { 79. Holds onto walls or } \\
\text { banisters (for example, } \\
\text { clings). }\end{array}$ & $\begin{array}{l}\text { 79. Segura-se nas paredes } \\
\text { ou corrimãos. }\end{array}$ & $\begin{array}{l}\text { 79. Supports self on walls } \\
\text { or handrails (for example, } \\
\text { grabs them). }\end{array}$ & $\begin{array}{l}\text { 79. Segura-se nas paredes } \\
\text { ou corrimãos (por } \\
\text { exemplo, agarra-se). }\end{array}$ \\
\hline $\begin{array}{l}\text { 83. Seeks opportunities to } \\
\text { fall without regard to } \\
\text { personal safety. }\end{array}$ & $\begin{array}{l}\text { 83. Procura cair sem se } \\
\text { preocupar com segurança } \\
\text { pessoal. }\end{array}$ & $\begin{array}{l}\text { 83. Tries to fall without } \\
\text { concern for own safety. }\end{array}$ & $\begin{array}{l}\text { 83. Busca oportunidades } \\
\text { para cair sem se preocupar } \\
\text { com segurança pessoal. }\end{array}$ \\
\hline
\end{tabular}


Quadro I. Avaliação da equivalência semântica, idiomática, conceitual e cultural entre o Sensory Profile original, a tradução, a retrotradução e a versão para aplicação (análise e comparação pelo comitê de especialistas) (conclusão)

\begin{tabular}{|c|c|c|c|}
\hline Original & Tradução & Retrotradução & Versão para aplicação \\
\hline 90. "On the go." & 90. “É muito ativo." & 90. "On the go," & 90. "Não para quieto." \\
\hline $\begin{array}{l}\text { 93. Rigid rituals in } \\
\text { personal hygiene. }\end{array}$ & $\begin{array}{l}\text { 93. Realiza rituais rígidos } \\
\text { de higiene pessoal. }\end{array}$ & $\begin{array}{l}\text { 93. Follows a rigid ritual } \\
\text { for personal hygiene. }\end{array}$ & $\begin{array}{l}\text { 93. Realiza rituais rígidos } \\
\text { de higiene pessoal (faz } \\
\text { sempre tudo da mesma } \\
\text { maneira). }\end{array}$ \\
\hline $\begin{array}{l}\text { 95. Doesn't perceive } \\
\text { body language or facial } \\
\text { expressions (for example, } \\
\text { unable to interpret). }\end{array}$ & $\begin{array}{l}\text { 95. Não compreende } \\
\text { linguagem corporal ou } \\
\text { expressões faciais (por } \\
\text { exemplo, não é capaz } \\
\text { de interpretar). }\end{array}$ & $\begin{array}{l}\text { 95. Does not understand } \\
\text { body language or facial } \\
\text { expressions (for example, } \\
\text { is not able to interpret). }\end{array}$ & $\begin{array}{l}\text { 95. Não compreende } \\
\text { gestos ou expressões } \\
\text { faciais (por exemplo, } \\
\text { não é capaz de } \\
\text { interpretar). }\end{array}$ \\
\hline $\begin{array}{l}\text { I05. Displays excessive } \\
\text { emotional outbursts } \\
\text { when unsuccessful at } \\
\text { a task. }\end{array}$ & $\begin{array}{l}\text { 105. Apresenta crises } \\
\text { emocionais excessivas } \\
\text { quando não é bem- } \\
\text {-sucedido em uma tarefa. }\end{array}$ & $\begin{array}{l}\text { 105. Has excessive } \\
\text { emotional crises when not } \\
\text { successful in a task. }\end{array}$ & $\begin{array}{l}\text { I05. Apresenta crises } \\
\text { emocionais excessivas } \\
\text { quando não consegue } \\
\text { realizar atividades. }\end{array}$ \\
\hline $\begin{array}{l}\text { I I } 7 . \text { Talks to self through } \\
\text { tasks. }\end{array}$ & $\begin{array}{l}\text { I I7. Fala consigo mesmo } \\
\text { durante tarefas. }\end{array}$ & $\begin{array}{l}\text { II } 7 . \text { Talks to self during } \\
\text { tasks. }\end{array}$ & $\begin{array}{l}\text { I I7. Fala consigo mesmo } \\
\text { durante atividades. }\end{array}$ \\
\hline $\begin{array}{l}\text { I25. Does not seem to } \\
\text { smell strong odors. }\end{array}$ & $\begin{array}{l}\text { I25. Não parece sentir } \\
\text { odores fortes. }\end{array}$ & $\begin{array}{l}\text { I25. Does not seem to } \\
\text { smell strong odors. }\end{array}$ & $\begin{array}{l}\text { I25. Não parece sentir } \\
\text { cheiros fortes. }\end{array}$ \\
\hline
\end{tabular}

Fonte: Elaborado pelas autoras.

\section{Verificação da aplicabilidade e consistência interna}

Os resultados dos dados coletados com a aplicação da versão traduzida e adaptada do instrumento estão demonstrados na Tabela 1. Os números de 1 a 50 representam os participantes. Na segunda e terceira colunas, respectivamente, estão registradas as idades das crianças avaliadas e a classificação. As letras de $\mathrm{A}$ até $\mathrm{N}$ representam as 14 categorias do Sensory Profile: processamento auditivo (A), processamento visual (B), processamento vestibular $(C)$, processamento tátil $(D)$, processamento multissensorial $(E)$, processamento oral sensorial $(F)$, processamento sensorial relacionado a resistência/tônus (G), modulação relacionada a posição corporal e movimento $(H)$, modulação de movimento que afeta o nível de atividade (I), modulação do estímulo sensorial que afeta as respostas emocionais (J), modulação de estímulo visual que afeta respostas emocionais e nível de atividade (L), respostas emocionais/sociais (M), efeitos comportamentais do processamento sensorial e itens que indicam limiares para resposta $(\mathrm{N})$. A partir do cálculo da variância das categorias e da variância dos escores brutos, obteve-se um alfa de Cronbach de 0,76 referente à pontuação total da escala. 


\begin{tabular}{|c|c|c|c|c|c|c|c|c|c|c|c|c|c|c|c|c|c|}
\hline & Idade & Classificação & A & B & C & $D$ & $E$ & $\mathrm{~F}$ & G & $\mathrm{H}$ & 1 & $J$ & $\mathrm{~K}$ & L & $M$ & $\mathrm{~N}$ & $\begin{array}{l}\text { Total por } \\
\text { criança }\end{array}$ \\
\hline I & 05 & D. TíP. & 30 & 44 & 46 & 81 & 31 & 50 & 45 & 27 & 23 & 11 & 12 & 59 & 22 & 12 & 493 \\
\hline 2 & 05 & D. TíP. & 31 & 38 & 50 & 80 & 30 & 50 & 45 & 45 & 22 & 20 & 16 & 72 & 26 & 15 & 540 \\
\hline 3 & 05 & D. TíP. & 33 & 34 & 38 & 70 & 30 & 41 & 32 & 38 & 21 & 16 & 14 & 73 & 23 & 13 & 476 \\
\hline 4 & 05 & D. TíP. & 22 & 36 & 42 & 72 & 25 & 43 & 41 & 42 & 26 & 13 & 12 & 63 & 22 & 10 & 469 \\
\hline 5 & 05 & D. TíP. & 32 & 35 & 39 & 61 & 28 & 31 & 40 & 40 & 20 & 14 & 12 & 65 & 25 & 14 & 456 \\
\hline 6 & 05 & D. TíP. & 27 & 33 & 36 & 63 & 20 & 38 & 35 & 34 & 19 & 18 & 13 & 49 & 17 & 13 & 415 \\
\hline 7 & 05 & D. TíP. & 27 & 36 & 43 & 67 & 24 & 47 & 38 & 42 & 25 & 18 & 13 & 53 & 21 & 13 & 467 \\
\hline 8 & 05 & TEA & 21 & 32 & 37 & 62 & 20 & 49 & 30 & 30 & 21 & 7 & 10 & 36 & 8 & 10 & 373 \\
\hline 9 & 06 & D. TíP. & 23 & 32 & 44 & 79 & 21 & 42 & 44 & 40 & 24 & 15 & 16 & 65 & 20 & 14 & 479 \\
\hline 10 & 06 & D. TíP. & 23 & 35 & 44 & 77 & 24 & 40 & 38 & 38 & 18 & 13 & 17 & 59 & 21 & 10 & 457 \\
\hline II & 06 & D. TíP. & 37 & 40 & 50 & 77 & 28 & 57 & 38 & 41 & 26 & 18 & 15 & 62 & 14 & 14 & 517 \\
\hline 12 & 06 & D. TíP. & 27 & 40 & 37 & 63 & 19 & 42 & 44 & 33 & 31 & 16 & 14 & 43 & 23 & 0 & 432 \\
\hline 13 & 06 & D. TíP. & 29 & 36 & 45 & 65 & 28 & 38 & 41 & 40 & 18 & 13 & 16 & 61 & 20 & 13 & 463 \\
\hline 14 & 06 & D. TíP. & 35 & 28 & 50 & 73 & 29 & 45 & 40 & 38 & 19 & 16 & 16 & 66 & 22 & 15 & 492 \\
\hline 15 & 06 & D. TíP. & 30 & 32 & 46 & 79 & 27 & 50 & 42 & 40 & 23 & 16 & 12 & 62 & 18 & 7 & 484 \\
\hline 16 & 06 & D. TíP. & 28 & 36 & 43 & 65 & 25 & 41 & 34 & 38 & 26 & 18 & 15 & 64 & 22 & 13 & 468 \\
\hline 17 & 06 & D. TíP. & 37 & 40 & 54 & 89 & 35 & 57 & 45 & 50 & 28 & 17 & 19 & 68 & 30 & 15 & 584 \\
\hline 18 & 06 & D. TíP. & 28 & 34 & 44 & 77 & 25 & 40 & 41 & 37 & 22 & 17 & 15 & 62 & 17 & 11 & 470 \\
\hline 19 & 06 & D. TíP. & 35 & 38 & 44 & 75 & 25 & 30 & 33 & 31 & 23 & 13 & 11 & 67 & 23 & 13 & 461 \\
\hline 20 & 06 & D. TíP. & 26 & 36 & 43 & 75 & 29 & 35 & 42 & 39 & 23 & 15 & 16 & 72 & 20 & 13 & 484 \\
\hline 21 & 06 & D. TíP. & 29 & 36 & 42 & 77 & 22 & 34 & 44 & 41 & 22 & 12 & 11 & 58 & 23 & 13 & 464 \\
\hline 22 & 06 & D. TíP. & 29 & 39 & 48 & 79 & 27 & 51 & 44 & 43 & 23 & 16 & 15 & 74 & 25 & 14 & 527 \\
\hline 23 & 06 & D. TíP. & 35 & 38 & 52 & 83 & 27 & 58 & 34 & 45 & 26 & 16 & 17 & 75 & 24 & 13 & 543 \\
\hline 24 & 06 & D. TíP. & 28 & 36 & 41 & 69 & 27 & 51 & 44 & 41 & 25 & 20 & 17 & 71 & 24 & 15 & 509 \\
\hline 25 & 06 & D. TíP. & 32 & 37 & 55 & 70 & 32 & 37 & 43 & 45 & 18 & 17 & 15 & 63 & 27 & 14 & 505 \\
\hline 26 & 06 & D. TíP. & 34 & 42 & 49 & 79 & 34 & 59 & 41 & 48 & 24 & 17 & 15 & 72 & 27 & 13 & 554 \\
\hline 27 & 06 & D. TíP. & 31 & 34 & 49 & 84 & 30 & 44 & 37 & 44 & 27 & 16 & 14 & 68 & 25 & 15 & 518 \\
\hline 28 & 07 & D. TíP. & 27 & 33 & 54 & 82 & 33 & 59 & 43 & 43 & 19 & 20 & 14 & 76 & 29 & 14 & 546 \\
\hline
\end{tabular}


Tabela I. Idade, classificação das crianças e pontuação por seção/categoria do Sensory Profile (conclusão)

\begin{tabular}{|c|c|c|c|c|c|c|c|c|c|c|c|c|c|c|c|c|c|}
\hline & Idade & Classificação & A & B & C & D & $\mathrm{E}$ & $F$ & G & $\mathrm{H}$ & I & J & K & $\mathrm{L}$ & M & $N$ & $\begin{array}{c}\text { Total por } \\
\text { criança }\end{array}$ \\
\hline 29 & 07 & D. TíP. & 31 & 39 & 53 & 88 & 31 & 46 & 44 & 47 & 27 & 19 & 19 & 73 & 26 & 15 & 558 \\
\hline 30 & 07 & D. TíP. & 33 & 34 & 54 & 75 & 21 & 56 & 44 & 32 & 24 & 15 & 15 & 66 & 25 & II & 505 \\
\hline 31 & 07 & D. TíP. & 33 & 29 & 44 & 69 & 27 & 48 & 43 & 44 & 23 & 18 & 17 & 70 & 23 & 10 & 498 \\
\hline 32 & 07 & D. TíP. & 32 & 36 & 48 & 84 & 30 & 47 & 44 & 43 & 27 & 15 & 14 & 71 & 26 & 14 & 594 \\
\hline 33 & 07 & D. TíP. & 33 & 38 & 45 & 71 & 25 & 42 & 38 & 39 & 25 & 13 & 14 & 55 & 20 & II & 531 \\
\hline 34 & 07 & S.DOWN & 24 & 27 & 37 & 78 & 21 & 44 & 24 & 33 & 18 & 11 & 13 & 57 & 15 & 13 & 415 \\
\hline 35 & 08 & D. TíP. & 20 & 29 & 45 & 66 & 26 & 39 & 41 & 39 & 16 & 13 & 13 & 59 & 19 & 13 & 438 \\
\hline 36 & 08 & D. TíP. & 37 & 42 & 54 & 84 & 33 & 57 & 44 & 47 & 23 & 17 & 16 & 73 & 26 & 14 & 567 \\
\hline 37 & 08 & D. TíP. & 33 & 32 & 48 & 79 & 32 & 52 & 39 & 45 & 27 & 19 & 16 & 63 & 20 & 14 & 519 \\
\hline 38 & 08 & D. TíP. & 40 & 45 & 55 & 86 & 34 & 56 & 44 & 47 & 29 & 19 & 19 & 76 & 29 & 15 & 493 \\
\hline 39 & 08 & D. TíP. & 34 & 41 & 50 & 76 & 31 & 31 & 44 & 40 & 24 & 11 & 17 & 53 & 27 & 14 & 467 \\
\hline 40 & 08 & D. TíP. & 25 & 41 & 42 & 65 & 23 & 41 & 45 & 36 & 22 & 14 & 14 & 61 & 24 & 14 & 515 \\
\hline 41 & 08 & D. TíP. & 27 & 28 & 36 & 62 & 22 & 43 & 28 & 32 & 22 & 10 & 12 & 56 & 18 & 9 & 492 \\
\hline 42 & 08 & D. TíP. & 29 & 37 & 45 & 69 & 28 & 43 & 34 & 40 & 28 & 18 & 15 & 68 & 24 & 14 & 541 \\
\hline 43 & 09 & D. TíP. & 34 & 44 & 53 & 87 & 34 & 60 & 45 & 50 & 31 & 20 & 20 & 82 & 30 & 15 & 500 \\
\hline 44 & 09 & D. TíP. & 34 & 35 & 44 & 72 & 27 & 45 & 45 & 44 & 23 & 17 & 15 & 75 & 25 & 14 & 405 \\
\hline 45 & 09 & D. TíP. & 34 & 36 & 49 & 77 & 29 & 50 & 29 & 39 & 13 & 16 & 15 & 61 & 23 & 13 & 468 \\
\hline 46 & 09 & D. TíP. & 40 & 45 & 55 & 90 & 35 & 60 & 45 & 47 & 23 & 20 & 18 & 79 & 30 & 15 & 605 \\
\hline 47 & 09 & S.DOWN & 25 & 34 & 46 & 79 & 28 & 49 & 39 & 49 & 18 & 8 & 12 & 66 & 15 & 13 & $48 I$ \\
\hline 48 & 10 & D. TíP. & 27 & 44 & 54 & 81 & 30 & 54 & 44 & 35 & 20 & 18 & 16 & 77 & 28 & 13 & 484 \\
\hline 49 & 10 & D. TíP. & 29 & 32 & 49 & 77 & 25 & 50 & 34 & 43 & 19 & 16 & 13 & 52 & 15 & 14 & 580 \\
\hline 50 & 10 & D. TíP. & 37 & 45 & 54 & 87 & 33 & 57 & 43 & 47 & 32 & 18 & 17 & 69 & 26 & 15 & 602 \\
\hline
\end{tabular}

D. TíP. = desenvolvimento típico; TEA = Transtorno do Espectro do Autismo; S.DOWN = síndrome de Down. Fonte: Elaborada pelas autoras.

\section{Discussão}

A inexistência no Brasil de instrumentos que avaliem as habilidades do processamento sensorial e seus efeitos no desempenho funcional e em tarefas diárias é reconhecida clínica, acadêmica e educacionalmente. Conforme descrito na introdução deste trabalho, instrumentos de avaliação sensorial como o Sensory Profile podem compor 
programas de rastreamento e protocolos de pesquisa e avaliação. Nesse sentido, este estudo, por meio da tradução e adaptação cultural do Sensory Profile, tem relevância acadêmica e social, pois viabiliza o desenvolvimento de pesquisas posteriores para a obtenção de evidências de validade desse instrumento, tornando possível sua utilização.

Adicionalmente, a revisão da literatura sobre a aplicação do Sensory Profile realçou a vasta aplicabilidade do instrumento em transtornos neuropsiquiátricos e em outras condições que afetam o processamento sensorial, enaltecendo a importância deste estudo.

A análise referente à pontuação total da escala revelou um valor Cronbach de 0,76, indicando que a consistência interna do instrumento é alta. $O$ valor mínimo de consistência interna aceitável para um instrumento conferido a partir do coeficiente do alfa de Cronbach, numa escala de 0 a 1, não é consensualmente estabelecido na literatura, mas há faixas descritas por Freitas e Rodrigues (2005), que foram utilizadas no presente estudo, a saber, alfa menor ou igual a 0,30 = consistência muito baixa; alfa de 0,30 a 0,60 = consistência baixa; alfa de 0,60 a 0,75 = consistência moderada; alfa de 0,75 a $0,90=$ consistência alta; e alfa maior que $0,90=$ consistência muito alta. Uma combinação de fatores, como o elevado grau de escolaridade dos participantes da amostra, oriundos de uma escola particular, a faixa etária das crianças avaliadas e o fato de a maioria delas apresentar desenvolvimento típico, pode ter contribuído para que o instrumento apresentasse uma consistência interna alta. A consistência interna do instrumento e as características dos participantes do nosso estudo são semelhantes às do conduzido por Neuman et al. (2004) para tradução e adaptação cultural do Sensory Profile do inglês para o hebraico. No que se refere à idade e escolaridade dos respondentes de uma amostra com 58 participantes, todas as crianças apresentaram desenvolvimento típico e valor de alfa de 0,7 para o total da escala. Posteriormente, Neuman (2006) demonstrou que os escores médios das crianças israelenses com desenvolvimento típico eram inferiores aos das norte-americanas, mas ainda dentro da faixa estabelecida para desenvolvimento típico. Uma limitação deste último estudo diz respeito à comparação dos dados obtidos na pesquisa com os da literatura, considerando a influência exercida pelas condições da aplicação do instrumento nos resultados. Entretanto, essas pesquisas arguem a favor de estudos com crianças com desenvolvimento típico para a tradução e adaptação do instrumento, bem como para evidências de validade com vistas a estabelecer um padrão de resposta da população brasileira. Além disso, nas pesquisas realizadas para o desenvolvimento e aperfeiçoamento do instrumento (Dunn \& Brown, 1997; Kientz \& Dunn, 1997; Ermer \& Dunn, 1998), aproximadamente $88 \%$ da amostra era composta por crianças com desenvolvimento típico e o restante distribuído entre TEA, síndrome do X Frágil, Transtorno Modular Sensorial e TDAH. Similarmente, no presente estudo, a maior parte da amostra constituiu-se de cuidadores de crianças com desenvolvimento típico, visando verificar a compreensibilidade da versão traduzida e adaptada. Em trabalho posterior, em que se busquem evidências de validade do instrumento, deverá ser incluída uma maior representatividade de cuidadores de crianças com desenvolvimento atípico. 
A metodologia utilizada neste estudo buscou a obtenção de uma versão para aplicação em português do Brasil adequadamente traduzida e culturalmente adaptada, mantendo equivalência com a versão original do instrumento. Considerando que o instrumento traduzido e adaptado mostrou uma consistência interna alta, isso indica que objetivo do estudo foi alcançado e que estudos posteriores poderão ser conduzidos a fim de buscar evidências de validade.

\section{Referências}

Alexandre, N. M. C., \& Guirardello, E. B. (2002). Adaptación cultural de instrumentos utilizados en salud ocupacional. Revista Panamericana de Salud Pública, 1, 109-111.

Almomani, F. M., Brown, C., Dahab, S. A., Almomani, M., \& Nadar, M. (2014). Crosscultural adaptation of the Adolescent/Adult Sensory Profile: establishing linguistic equivalency and psycometric properties of the Arabic version. Disability and Rehabilitation, 36, 765-770.

Ashburner, J., Ziviani, J., \& Rodger, S. (2008). Sensory processing and classroom emotional, behavioral and educational outcomes in children with Autism Spectrum Disorder. American Journal of Occupational Therapy, 62, 564-573.

Ayres, A. J. (1972). Sensory integration and learning disorders. Los Angeles: Western Psychological Services.

Ayres, A. J. (1989). Sensory Integration and Praxis Tests: SIFT manual. Los Angeles: Western Psychological Services.

Brown, N. B., \& Dunn, W. (2010). Relationship between context and sensory processing in children with autism. American Journal of Occupational Therapy, 64, 474-483.

Brown, T., Leo, M., \& Austin, D. W. (2008). Discriminant validity of the Sensory Profile in Australian children with Autism Spectrum Disorder. Physical \& Occupational Therapy in Pediatrics, 28, 253-266.

Case-Smith, J., Weaver, L. L., \& Fristad, M. A. (2015). A systematic review of sensory processing interventions for children with Autism Spectrum Disordes. Autism, 19(2), 133-148.

Chung, J. C. (2006). Measuring sensory processing patterns of older Chinese people: psychometric validation of the Adult Sensory Profile. Aging \& Mental Health, 10, 648-655.

DeGangi, G. A., \& Stanley, S. I. (1989). The development of sensory functions in infants. Physical \& Occupational Therapy in Pediatrics, 8(4), 21-33. 
Dunn, W. (1999a). Caregiver Questionnaire - Sensory Profile. San Antonio, TX: Pearson.

Dunn, W. (1999b). User's Manual - Sensory Profile. San Antonio, TX: Pearson.

Dunn, W. (2002). The infant toddler Sensory Profile. San Antonio, TX: Psychological Corporation.

Dunn, W. (2006). Sensory Profile School Companion: User's manual. San Antonio, TX: Psychological Corporation.

Dunn, W., \& Brown, C. (1997). Factor analysis on the Sensory Profile from a national sample of children without disabilities. American Journal of Occupational Therapy, 51(7), 490-495.

Engel-Yeger, B. (2010). The applicability of the Short Sensory Profile for screening sensory processing disorders among Israeli children. International Journal of Rehabilitation Research, 4, 311-318.

Engel-Yeger, B., Almog, M., \& Kessel, A. (2014). The Sensory Profile of children with asthma. Acta Pediatric, 11, 490-494.

Engel-Yeger, B., Habib-Mazawi, S., Parush, S., Rozenman, D., Kessel, A., \& Shani-Adir, A. (2007). The Sensory Profile of children with atopic dermatitis as determined by the Sensory Profile Questionnaire. Journal of the American Academy of Dermatology, 4, 610-615.

Engel-Yeger, B., Hardal-Nasser, R., \& Gal, E. (2011). Sensory processing dysfunctions as expressed among children with different severities of intellectual developmental disabilities. Research in Developmental Disabilities, 5, 1770-1775.

Ermer, J., \& Dunn, W. (1998). The Sensory Profile: a discriminant analysis of children with and without disabilities. American Journal of Occupational Therapy, 52(4), 283-290.

Freitas, A. L. P., \& Rodrigues, S. G. (2005). A avaliação da confiabilidade de questionário: uma análise utilizando o coeficiente alfa de Cronbach. XII Simpósio de Engenharia de Produção, Bauru, SP, Brasil. DOI: 10.13140/2.3075.6808.

Ganapathy, S. U., \& Priyadarshini, S. (2014). Standardization of Tamil version of Short Sensory Profile. International Journal of Pharma and Bio Sciences, 5, 260-266.

Javitt, D. C. (2009). Sensory processing in schizophrenia: neither simple nor intact. Schizophrenia Bulletin, 35(6), 1059-1064.

Kientz, M. A., \& Dunn, W. (1997). Comparison of the performance of children with and without autism on the Sensory Profile. American Journal of Occupational Therapy, 51(7), 530-537.

Koziol L. F., \& Budding, D. (2012). ADHD and sensory processing disorders: placing the diagnostic issues in context. Applied Neuropsychology Child, 1(2), 137-144. 
Neuman, A. (2006). Patterns of response to sensory stimuli encountered in daily activities: a comparison between 3-10-year-old Israeli and American children without disabilities. Occupational Therapy International, 13(2), 79-99.

Neuman, A., Greenberg, D. F., Labovitz, D. R., \& Suzuki, L. A. (2004). Cross-cultural adaptation of the Sensory Profile: establishing linguistic equivalency of the Hebrew version. Occupational Therapy International, 11(3), 112-130.

Sampieri, R. H., Collado, C. F., \& Lucio, P. B. (2006). Metodologia de pesquisa (3a ed.). São Paulo: McGraw-Hill.

Tomchek, S. D., \& Dunn, W. (2007). Sensory processing in children with and without autism: a comparative study using the Short Sensory Profile. American Journal of Occupational Therapy, 61(2), 190-200.

Submissão: 9.6.2015

Aceitação: 10.8.2015 